\title{
Vírus do tumor mamário de camundongo (MMTV) e câncer de mama - associação de causalidade determinante no desfecho clínico
}

\author{
Mouse mammary tumor virus (MMTV) and breast cancer - determinant causality association in \\ clinical outcome
}

Virus del tumor mamario de ratón (MMTV) y cáncer de mama - associación de causalidad determinante en el resultado clínico

Recebido: 02/06/2021 | Revisado: 09/06/2021 | Aceito: 10/06/2021 | Publicado: 23/06/2021

\author{
Guilherme Guimarães Leal \\ ORCID: https://orcid.org/0000-0001-9165-4330 \\ Universidade de Ribeirão Preto, Brasil \\ E-mail: guileag111@gmail.com \\ Helen Figueiredo Fumagalli \\ ORCID: https://orcid.org/0000-0002-4607-8976 \\ Universidade de Ribeirão Preto, Brasil \\ E-mail: hfumagalli@unaerp.br
}

\begin{abstract}
Resumo
Objetiva-se alertar sobre o possível papel de infecções virais causadas pelo vírus do tumor mamário de camundongo (MMTV) no desenvolvimento e progressão do câncer (CA) de mama, enfatizando a prevalência do achado do vírus nessa patologia, e sua ação determinante no desfecho clínico carcinogênico. Sendo que, neste trabalho foi realizado um levantamento bibliográfico, procurando por produções científicas publicadas nos periódicos PubMed, LILACS e SciELO. A partir desse levantamento, foi realizada uma revisão sistematizada, sendo utilizado os artigos científicos dos últimos 5 anos, nas versões português, espanhol e inglês. Geralmente, os estudos realizados com Reação em Cadeia da Polimerase (PCR) e técnicas de hibridização foram levados em consideração. Assim, foi observada uma prevalência da carcinogênese mamária induzida pelo vírus do tumor mamário de camundongo (média de 31,6\%), quando comparada a ocasião em que o achado do vírus foi positivo, mas não induzindo tal patologia. Além de que, quando o achado do vírus foi positivo induzindo a carcinogênese, foi evidenciado a inibição da metástase linfonodal. Dessa forma, o vírus do tumor mamário de camundongo se consolida como um fator de risco para o desenvolvimento do CA de mama. Ademais, o achado do vírus no câncer de mama foi associado a um melhor prognóstico, por inibir a disseminação sanguínea/linfática das células tumorais. Portanto, a partir possibilidade de que as células tumorais possam exibir antígenos virais, estabelece-se um alvo antigênico em potencial, com a promessa de desenvolvimento de tratamentos preventivos para o câncer de mama.
\end{abstract}

Palavras-chave: Câncer de mama; Infecções virais; MMTV; Oncogenes.

\begin{abstract}
The objective is to alert about the possible role of viral infections caused by the mouse mammary tumor virus (MMTV) in the development and progression of breast cancer (BC), emphasizing the prevalence of the virus finding in this pathology, and its determinant action in the carcinogenic clinical outcome. In this work, a bibliographical survey was carried out, searching for scientific productions published in PubMed, LILACS and SciELO journals. From this survey, a systematic review was carried out, using scientific articles from the last 5 years, in Portuguese, Spanish and English versions. Generally, studies carried out with Polymerase Chain Reaction (PCR) and hybridization techniques were taken into consideration. Thus, a prevalence of mammary carcinogenesis induced by the mouse mammary tumor virus (mean $31.6 \%$ ) was observed when compared to the occasion when the virus finding was positive but not inducing such pathology. In addition, when the virus was positive, inducing carcinogenesis, the inhibition of lymph node metastasis was evidenced. Thus, the mouse mammary tumor virus is consolidated as a risk factor for the development of breast cancer. Moreover, the finding of the virus in breast cancer was associated with a better prognosis, by inhibiting blood/lymphatic dissemination of tumor cells. Therefore, from the possibility that tumor cells may display viral antigens, a potential antigenic target is established, with the promise of developing preventive treatments for breast cancer.
\end{abstract}

Keywords: Breast cancer; Viral infections; MMTV; Oncogenes. 


\begin{abstract}
Resumen
Su objetivo es alertar sobre el posible papel de las infecciones virales causadas por el virus del tumor mamario de ratón (MMTV) en el desarrollo y la progresión del cáncer de mama (CM), destacando la prevalencia del hallazgo del virus en esta patología, y su acción determinante en el resultado clínico carcinogénico. En este trabajo se realizó un relevamiento bibliográfico, buscando producciones científicas publicadas en las revistas PubMed, LILACS y SciELO. A partir de esta encuesta, se realizó una revisión sistemática, utilizando artículos científicos de los últimos 5 años, en versiones en portugués, español e inglés. En general, se tomaron en consideración los estudios realizados con técnicas de reacción en cadena de la polimerasa (PCR) y de hibridación. Así, se observó una prevalencia de carcinogénesis mamaria inducida por el virus del tumor mamario de ratón (media del 31,6\%) en comparación con la ocasión en que el hallazgo del virus fue positivo, pero no indujo dicha patología. Además, cuando el hallazgo del virus fue positivo, induciendo la carcinogénesis, se evidenció la inhibición de la metástasis en los ganglios linfáticos. Así, el virus del tumor mamario del ratón se consolida como un factor de riesgo para el desarrollo del CA de mama. Además, el hallazgo del virus en el cáncer de mama se asoció a un mejor pronóstico, al inhibir la diseminación sanguínea/linfática de las células tumorales. Por lo tanto, partiendo de la posibilidad de que las células tumorales puedan mostrar antígenos virales, se establece una potencial diana antigénica, con la promesa de desarrollar tratamientos preventivos para el cáncer de mama.
\end{abstract}

Palabras clave: Cáncer de mama; Infecciones virales; MMTV; Oncogenes.

\title{
1. Introdução
}

O câncer de mama é o tipo de câncer mais comum entre as mulheres no mundo e no Brasil, depois do de pele não melanoma, respondendo por cerca de $25 \%$ dos casos novos a cada ano. Também acomete homens, porém é raro, representando apenas $1 \%$ do total de casos da doença. Refere-se a um tumor maligno, formado pelo crescimento de células de maneira desordenada, e desenvolvimento de um ou mais nódulos na mama.

Embora desconhecidas as causas para a patogênese do câncer (CA) de mama, diversas variáveis atuam como fatores de risco, tais como: (1) Sexo feminino; (2) Idade, como sendo um dos mais importantes, na medida em que há o acúmulo de exposições ao longo da vida e alterações biológicas advindas do envelhecimento, sendo o CA prevalente em mulheres a partir dos 50 anos; (3) Predisposição genética, geralmente relacionada à presença de mutações em determinados genes transmitidos na família, como BRCA1 e BRCA2. (4) Fatores comportamentais e/ou ambientais; (5) Consumo de álcool; (6) Tabagismo; (7) Sobrepeso e obesidade; (8) Exposição à radiação ionizante (advindos de radioterapia e exames de imagem, como mamografia e tomografia computadorizada), sendo proporcional à dose e à frequência; (9) Fatores endócrinos e reprodutivos, sendo relacionados com a produção de estrógeno pelo organismo ou seu consumo continuado (medicamentos - contraceptivos ou terapia de reposição hormonal pós-menopausa); (10) Menarca precoce (menor que 12 anos); (11) Menopausa tardia (após os 55 anos); (12) Primeira gravidez após os 30 anos; (13) Nuliparidade.

De modo interessante, alguns fatores comportamentais agem como protetores, ajudando a reduzir o risco de câncer de mama, dentre esses, podemos citar: (1) Amamentação - possivelmente em razão de mecanismos biológicos, como a forte exfoliação do tecido mamário, alterações na estrutura da mama, intensa apoptose epitelial ao final da amamentação, e redução do tempo de exposição da lactente ao estrogênio e outros hormônios durante a amenorreia; (2) Prática de atividade física na menopausa, geralmente relacionada a redução da gordura corporal, (3) redução dos níveis circulantes de estrogênio, (4) redução da resistência à insulina e (5) redução da inflamação. Somado a isso, se na pré-menopausa, a (6) atividade física for de intensidade vigorosa (nadar, correr, ciclismo) há também um provável efeito protetor no que tange o risco ao CA.

Entretanto, os fatores de risco descritos anteriormente não são únicos, uma vez que evidências recentes demonstraram que alguns vírus agem como indutores do carcinoma de mama, dentre estes: vírus Epstein-Barr, vírus do papiloma humano, vírus do tumor mamário de camundongo e vírus da leucemia bovina.

Neste contexto, o MMTV, conhecido por causar CA de mama em camundongos por mutagênese de inserção, tem chamado a atenção de alguns grupos de pesquisadores, uma vez que sequências de seu genoma têm sido isoladas de amostras de tecido de CA de mama em humanos, corroborando com a teoria da origem viral de alguns casos. A partícula viral citada pertence 
à família Retroviridae, e ao gênero Lentivirus. Assim, a identificação da sequência do MMTV, retrovírus tipo B, tem suportado a teoria da origem viral no câncer de mama.

Além disso, é válido ressaltar que o câncer de mama pode ser estratificado pela expressão de marcadores moleculares, como receptores de estrogênio (RE), receptores de progesterona (RP) e superexpressão ou amplificação do receptor do fator de crescimento epidérmico humano 2 (HER2), classificando a doença em quatro subtipos moleculares: A luminal (LA; ER/PR + HER2-), Luminal-B HER2-positivo (LB; ER/PR + HER2+), HER2-enriquecido (HER2; ER-PR-HER2+) e triplo-negativo (TN; ER-PR-HER2-). Esses subtipos têm um impacto direto no prognóstico e na resposta terapêutica dos pacientes (Pereira, et al., 2020).

Assim, o presente trabalho teve como objetivos: alertar sobre o CA de mama e seus fatores de risco e protetores já estabelecidos; relatar estudos que envolvem o possível papel de infecções virais causadas pelo vírus MMTV no desenvolvimento e progressão do câncer de mama, enfatizando a prevalência do achado do vírus no câncer de mama; associar a presença do vírus do tumor mamário de camundongo como determinante no desfecho clínico carcinogênico; dar suporte a ações preventivas e terapêuticas a partir da etiologia do CA de mama induzido por um agente viral.

\section{Metodologia}

O presente trabalho trata-se de uma revisão bibliográfica voltada à metanálise, onde procurou-se sintetizar objetivamente os achados de alguns trabalhos clínicos, tal como descrito por Vieira \& Hosne (2015). Para tanto, foram realizadas buscas de dados bibliográficos em três plataformas: PubMed, LILACS e SciELO. A partir deste levantamento, realizou-se uma revisão bibliográfica, sendo utilizado os artigos científicos dos últimos cinco anos, nas línguas portuguesa, inglesa e espanhola.

Utilizou-se como critério de inclusão aqueles artigos que apontaram o uso de técnicas moleculares pré-definidas para detecção do RNA viral:

1. Reação em Cadeia da Polimerase (PCR).

2. Técnicas de hibridização.

Esta última com aplicação justificada após detecção ou não do RNA viral por PCR, porque apesar da PCR ser de alta sensibilidade, a proporção de ácido nucleico viral em células hospedeiras infectadas é muito pequena, podendo obter resultados inconsistentes (falsos-negativos). Assim, a partir da utilização de uma sonda para detecção do sequenciamento de nucleotídeos específicos nos tecidos das amostras, há a confirmação do resultado obtido na técnica de PCR.

Por fim, foram excluídas publicações cuja veracidade não foi devidamente comprovada, bem como trabalhos datados de mais de 5 anos.

\section{Resultados}

Um estudo publicado por Lawson, et al. (2018) foi analisado, os quais observaram os resultados obtidos de 16 estudos de casos-controle, baseados em técnicas de PCR e/ou hibridização in situ. Neste estudo, foram pesquisados carcinomas em estágios avançados (invasivos) e carcinomas ductais in situ. Sendo que, foi verificada a prevalência do MMTV positivo induzindo a carcinogênese mamária no carcinoma em um estágio mais avançado (invasivo), enquanto que o carcinoma ductal in situ foi também levado em consideração, mas somente quando não induzido pelo vírus MMTV (Quadro 1). 
Quadro 1. Análise do achado de sequências de MMTV em tecidos de mama cancerosos e comparação de espécimes de mama não cancerosos.

\begin{tabular}{|c|c|c|}
\hline & $\begin{array}{l}\text { MMTV POSITIVO INDUZIDO A } \\
\text { CARCINOGÊNESE MAMÁRIA }\end{array}$ & $\begin{array}{c}\text { MMTV POSITIVO NÃO } \\
\text { INDUZINDO A } \\
\text { CARCINOGÊNESE MAMÁRIA }\end{array}$ \\
\hline Ford, et al., 2004 & $45 / 144(31 \%)$ & $0 / 20(0 \%)$ \\
\hline Etkind, et al., 2000 & $27 / 73(37 \%)$ & $0 / 35(0 \%)$ \\
\hline Melana, et al., 2001 & $32 / 106(30 \%)$ & $1 / 106(1 \%)$ \\
\hline Melana, et al., 2002 & $23 / 74(31 \%)$ & $1 / 10(10 \%)$ \\
\hline Ford, et al., 2003 & $14 / 26(54 \%)$ & $2 / 111(2 \%)$ \\
\hline Wang, et al., 1995 & $121 / 314(38,5 \%)$ & $2 / 107(2 \%)$ \\
\hline Zammarchi, et al., 2006 & $13 / 43(30 \%)$ & $1 / 8(12.5 \%)$ \\
\hline Bindra, et al., 2007 & $0 / 18(0 \%)$ & $0 / 11(0 \%)$ \\
\hline Glenn, et al., 2012 & $39 / 50(78 \%)$ & $13 / 40(33 \%)$ \\
\hline Shariatpanahi, et al., 2017 & $19 / 59(32 \%)$ & $3 / 59(5 \%)$ \\
\hline Hachana, et al., 2008 & $17 / 122(14 \%)$ & $0 / 122(0 \%)$ \\
\hline Slaoui, et al., 2014 & $24 / 57(42 \%)$ & $6 / 18(33 \%)$ \\
\hline Cedro-Tanda, et al., 2014 & $57 / 458(12 \%)$ & $72 / 458(16 \%)$ \\
\hline Reza, et al., 2015 & $12 / 100(12 \%)$ & $0 / 100(0 \%)$ \\
\hline Naushad, et al., 2017 & $83 / 250(29 \%)$ & $0 / 15(0 \%)$ \\
\hline Mazzanti, et al., 2011 & $7 / 20(35 \%)$ & $0 / 20(0 \%)$ \\
\hline MÉDIA & $31,6 \%$ & $7,15 \%$ \\
\hline
\end{tabular}

Fonte: Adaptado de Lawson, et al. (2018).

Assim, por meio deste quadro, nota-se a prevalência da carcinogênese mamária induzida pelo vírus do tumor mamário de camundongo (média de 31,6\%), quando comparada a ocasião em que o achado do vírus foi positivo, mas não induzindo a carcinogênese (média de 7,15\%).

Outro estudo, publicado por Pereira, et al. (2020), analisou o achado de sequências exógenas do MMTV em 18,9\% (41 das 217 amostras) dos tecidos tumorais analisados. Sendo que, tumores que expressam HER2 mostraram uma maior prevalência de MMTV-like env no tecido tumoral, com a maior prevalência sendo observada em tumores do subtipo HER2-enriquecido (5/13 - 38,5\%), seguido por aqueles do subtipo Luminal B (6/24 - 25\%) e subtipo Luminal A (27/139 - 19,4\%). Tumores TN, por outro lado, mostraram a menor prevalência de MMTV-like env, com apenas uma das 26 amostras (3,8\%) sendo positiva.

Também foi analisado a presença de sequências exógenas de MMTV em dois grupos representativos de câncer de mama: carcinoma de mama hereditário e carcinoma esporádico da mama, excluindo qualquer contaminação por material murino e retroviral, e beta-retrovírus endógenos. Assim, Naccarato, et al. (2019) evidenciou que 30,3\% de 56 dos casos de carcinoma esporádico de mama continham sequências MMTV, contra 4,2\% de 47 de carcinoma de mama hereditário (p < 0,001). Casos positivos para sequências virais mostraram a presença de p14, peptídeo sinal do precursor do envelope MMTV.

Tendo em vista que o CA de mama pode ser um tumor hormônio-dependente, diversos estudos têm sido desenvolvidos a fim de determinar a influência hormonal na ação do MMTV. Tal fato baseia-se na prevalência de partículas retrovirais semelhantes ao MMTV (MMTVLS) em mulheres com CA de mama gestacional (câncer que ocorre durante a gravidez ou 12 meses após o parto) ser muito maior (62\%), em comparação com o CA de mama em mulheres não grávidas (30-38\%) (Hernández, et al., 2016). 


\section{Discussão}

Em camundongos, a transmissão viral pode ocorrer através do leite materno, ou os filhotes podem herdar o vírus diretamente da mãe. Porém, em humanos, apesar de não se saber ao certo a via de transmissão, a infecção pelo MMTV é aparentemente adquirida de uma espécie de camundongo (Mus domesticus). De modo interessante, observa-se maior prevalência da infecção viral em áreas geográficas com acentuada exposição a essa espécie de rato doméstico, principalmente em países ocidentais (30-40\%) e asiáticos (10-20\%) (Stewart, et al., 2000).

Nesse cenário, notou-se que a célula humana hospedeira, por não apresentar oncogenes, desenvolve sua capacidade oncogênica indiretamente, por mutagênese insercional. Assim, durante a replicação, há integração do DNA pró-viral no genoma alvo, próximo a um ou mais dos proto-oncogenes (Wnt-1, FgF e Notch), o que resulta na ativação dos oncogenes envolvidos e na expressão desregulada de fatores de crescimento celular nas células infectadas.

Além disso, por ser um retrovírus, pode aumentar a expressão de membros do cluster oncogênico miRNA da célula hospedeira infectada, em especial mi-17-92. Assim, essas pequenas moléculas de RNA (RNA de interferência, não codificantes) agem como silenciadores pós-transcricionais, pareando-se com ácidos nucleicos específicos e, consequentemente, regulando sua estabilidade e atividade traducional. Dessa forma, os genes da célula hospedeira tornam-se oncogênicos pela inclusão de bases nitrogenadas virais em seu genoma, desencadeando a continuidade do ciclo celular, mesmo sem estímulo evidente.

Sendo que, nos subtipos LB e HER2-enriquecido, Pereira, et al. (2020) observaram correlações entre a presença de MMTV no tecido tumoral e parâmetros clínico-patológicos indicativos de melhor prognóstico, como menor tamanho do tumor, menor estadiamento TNM e menor frequência de metástase linfonodal (primeiro evento grave na carcinogênese mamária, associado a um prognóstico desfavorável).

Assim, foi demonstrado que o MMTV não codifica miRNAs, mas altera a expressão da rede de miRNA do hospedeiro. Dessa forma, regula positivamente a expressão de miRNAs oncogênicos do cluster miR-17-92, associado a câncer de mama, enquanto regula negativamente a expressão de miR-10b-5p, que está associado à promoção de metástases. Esses resultados indicam um provável mecanismo pelo qual o MMTV pode promover a carcinogênese, enquanto inibe a metástase. Tal evidência pode ser consistente com os resultados paradoxais encontrados no subgrupo LA, no qual o MMTV-like foi positivamente correlacionado com o índice de proliferação, mas negativamente correlacionado com metástase linfonodal. Outro possível mecanismo que explica a associação do tipo MMTV com melhor prognóstico, pode envolver a ativação de respostas imunes antivirais no microambiente tumoral. De fato, o câncer de mama mostra expressão aumentada de genes relacionados à inflamação e infecção viral, como interferons (IFN). A resposta imune fornecida pelos IFNs tipo I, que são classicamente ativados durante infecções virais, mostrou ser um fator supressor importante no processo metastático. Além disso, vários estudos têm mostrado que tanto a expressão de retrovírus endógenos quanto a infecção com vírus exógenos, indicam imunogenicidade aumentada e melhor prognóstico geral em vários tipos de câncer (Pereira, et al., 2020).

Ademais, foi proposto que as proteínas do envelope viral do MMTV (gp 36 e gp 52) também estariam envolvidas na tumorigênese, na medida em que induziriam a transformação de células mamárias em neoplásicas - capacidade dos betaretrovírus em transformarem diretamente as células.

\section{Conclusão}

Apesar da ação viral no desenvolvimento de CA de mama ainda ser um tema controverso, a revisão demonstrou ser possível a influência do vírus MMTV na carcinogênese mamária, evidenciada pela prevalência significativamente maior em tecidos de câncer de mama em comparação com tecidos de controle benignos ou normais. 
Tais evidências apresentaram variáveis geográficas, de acordo com a maior ou menor exposição da espécie de camundongo Mus domesticus, transmissor do vírus MMTV. Dessa forma, há um alvo antigênico em potencial, com a possibilidade de desenvolvimento de abordagens preventivas para uma doença tão complexa e heterogênea.

No entanto, as sequências de MMTV que induziram o CA de mama também foram associadas a melhores parâmetros de prognóstico em subtipos específicos. Tal evidência fortalece ainda mais o seu potencial como um marcador valioso para o prognóstico de câncer de mama, além de ser um fator de risco para tal.

Além disso, o estudo favorece a confiabilidade dos dados que sustentam a associação entre MMTV e carcinoma esporádico de mama, devido a maior prevalência de sequências exógenas de MMTV nesses casos de câncer de mama, em comparação com o carcinoma de mama hereditário. Esse resultado era esperado, com base no fato do carcinoma de mama hereditário possuir etiologia genética específica, não necessitando da ação de um agente viral carcinogênico.

Em meio ao cenário apresentado, faz-se necessário a ampliação massiva de pesquisas clínicas laboratoriais, que possam dar maior suporte à prevalência do MMTV nas amostras de biópsias de CA de mama, o que estabeleceria uma melhor relação entre o gene aqui apontado e sua fonte. Também se fará interessante a abordagem do gene como alvo de pesquisa preditiva em mulheres convíveres da espécie de $M$. domesticus, com o intuito de promover o diagnóstico precoce do CA de mama.

\section{Referências}

Bindra, A., Muradrasoli, S., Kisekka, R., Nordgren, H., Wärnberg, F. \& Blomberg, J. (2007). Search for DNA of exogenous mouse mammary tumor virusrelated virus in human breast cancer samples. Journal of General Virology, 88(6), 1806-1809. Apud Lawson, J. S. et al., 2018.

Cedro-Tanda, A., Córdova-Solis, A., Juárez-Cedillo, T., Pina-Jiménez, E., Henández-Caballero, M. E., Moctezuma-Meza, C., Castelazo-Rico, G., GómezDelgado, A., Monsalvo-Reyes, A. C., Salamanca-Gómez, F. A., Arenas-Aranda, D. J. \& García-Hernández, N. (2014). Prevalence of HMTV in breast carcinomas and unaffected tissue from Mexican women. BMC Cancer, 14. Apud Lawson, J. S. et al., 2018.

Etkind, P., Du, J., Khan, A., Pillitteri, J. \& Wiernik, P. H. (2000). Mouse mammary tumor virus-like env gene sequences in human breast tumors and in a lymphoma of a breast cancer patient. Clinical Cancer Research, 6, 1273-1278. Apud Lawson, J. S. et al., 2018.

Ford, C. E., Faedo, M. \& Rawlinson, W. D. (2004). Mouse mammary tumor virus-like RNA transcripts and DNA are found in affected cells of human breast cancer. Clinical Cancer Research, 10(21), 7284-7289. Apud Lawson, J. S. et al., 2018.

Ford, C. E., Tran, D., Deng, Y., Ta, V. T., Rawlinson, W. D. \& Lawson, J. S (2003). Mouse Mammary Tumor Virus-like Gene Sequences in Breast Tumors of Australian and Vietnamese Women. Clinical Cancer Research, 9(3), 1118-1120. Apud Lawson, J. S. et al., 2018.

Glenn, W. K., Heng, B., Delprado, W., Iacopetta, B., Whitaker, N. J. \& Lawson, J. S. (2012). Epstein-Barr virus, human papillomavirus and mouse mammary tumour virus as multiple viruses in breast cancer. PLoS One, 7(11). Apud Lawson, J. S. et al., 2018.

Hachana, M., Trimeche, M., Ziadi, S., Amara, K., Gaddas, N., Mokni, M. \& Korbi, S. (2008). Prevalence and characteristics of the MMTV-like associated breast carcinomas in Tunisia. Cancer Letters, 271(2), 222-230. Apud Lawson, J. S. et al., 2018.

Hernández, M. L. A. R., Menéndez, M. H. \& Noda, M. F. (2016). Infecciones virales, posible factor de riesgo en cáncer de mama. Revista Cubana de Obstetricia y Ginecología, 42(3), 412-424.

Lawson, J. S., Glenn, W. K. \& Whitaker, N. J. (2016). Human Papilloma Viruses and Breast Cancer - Assessment of Causality. Frontiers in Oncology, 6.

Lawson, J. S., Glenn, W. K., Salmons, B., Ye, Y., Heng, B., Moody, P., Johal, H., Rawlinson, W. D., Delprado, W., Lutze-Mann, L. \& Whitaker, N. J. (2010). Mouse mammary tumor virus-like sequences in human breast cancer. Cancer Research, 70(9), 3576-3585. Apud Lawson, J. S. et al., 2018.

Lawson, J. S., Salmons, B. \& Glenn, W. K. (2018). Oncogenic Viruses and Breast Cancer: Mouse Mammary Tumor virus (MMTV), Bovine Leukemia Virus (BLV), Human Papilloma Virus (HPV), and Epstein-Barr Virus (EBV). Frontiers in Oncology, 8(1).

Lehrer, S. \& Rheinstein, P. H. (2019). The Virology of Breast Cancer: Viruses as the Potential Causative Agents of Breast Tumorigenesis. Discov Med, 27(148), $163-166$.

Mazzanti, C. M., Hamad, M. A., Fanelli, G., Scatena, C., Zammarchi, F., Zavaglia, K., Lessi, F., Pistello, M., Naccarato, A. G. \& Bevilacqua, G. (2011). A mouse mammary tumor virus env-like exogenous sequence is strictly related to progression of human sporadic breast carcinoma. The American Journal of Pathology, 179(4), 2083-2090. Apud Lawson, J. S. et al., 2018.

Melana, S. M., Holland, J. F. \& Pogo, B. G. (2001). Search for Mouse Mammary Tumor Virus-like env Sequences in Cancer and Normal Breast from the Same Individuals. Clinical Cancer Research, 7(2), 283-284. Apud Lawson, J. S. et al., 2018.

Melana, S. M., Picconi, M. A., Rossi, C., Mural, J., Alonio, L. V., Teyssié, A., Holland, J. F. \& Pogo, B. G. (2002). Detection of murine mammary tumor virus (MMTV) env gene-like sequences in breast cancer from Argentine patients. Medicina (B Aires), 62(4), 323-327. Apud Lawson, J. S. et al., 2018. 
Naccarato, A. G., Lessi, F., Zavaglia, K., Scatena, C., Hamad, M. A. A. H., Aretini, P., Menicagli, M., Roncella, M., Ghilli, M., Caligo, M. A., Mazzanti, C. M. \& Bevilacqua, G. (2019). Mouse mammary tumor virus (MMTV) - like exogenous sequences are associated with sporadic but not hereditary human breast carcinoma. Aging, 11(17), 7236-7241.

Nartey, T., Mazzanti, C. M., Melana, S., Glenn, W. K., Bevilacqua, G., Holland, J. F., Whitaker, N. J., Lawson, J. S. \& Pogo, B. G-T. (2017). Mouse mammary tumor-like virus (MMTV) is present in human breast tissue before development of virally associated breast cancer. Infectious Agents and Cancer, 12(1). Apud Lehrer, S. et al., 2019

Naushad, W., Surriya, O. \& Sadia, H. (2017). Prevalence of EBV, HPV and MMTV in Pakistani breast cancer patients: a possible etiological role of viruses in breast cancer. Infection, Genetics and Evolution, 54, 230-237. Apud Lawson, J. S. et al., 2018.

Pereira, N. D. S., Vitiello, G. A. F., Banin-Hirata, B. K., Fernandes, G. S. A., Salles, M. J. S., Amarante, M. K. \& Watanabe, M. A. E. (2020). Mouse Mammary Tumor Virus (MMTV)-Like env Sequence in Brazilian Breast Cancer Samples: Implications in Clinicopathological Parameters in Molecular Subtypes. International Journal of Environmental Research and Public Health, 17(24).

Reza, M. A., Reza, M. H., Mahdiyeh, L., Mehdi, F. \& Hamid, Z. N. (2015). Evaluation frequency of Merkel Cell Polyoma, Epstein-Barr and mouse mammary tumor viruses in patients with breast cancer in Kerman, southeast of Iran. Asian Pac J Cancer Prev, 16(16), 7351-7357. Apud Lawson, J. S. et al., 2018.

Shariatpanahi, S., Farahani, N., Salehi, A. R. \& Salehi, R. (2017). High prevalence of mouse mammary tumor virus-like gene sequences in breast cancer samples of Iranian women. Nucleosides Nucleotides Nucleic Acids, 36(10), 621-630. Apud Lawson, J. S. et al., 2018.

Slaoui, M., Mzibri, M. E., Razine, R., Qmichou, Z., Attaleb, M. \& Amrani, M. (2014). Detection of MMTV-Like sequences in Moroccan breast cancer cases. Infectious Agents and Cancer, 9(37). Apud Lawson, J. S. et al., 2018.

Stewart, T. H. M., Sage, R. D., Stewart, A. F. R \& Cameron, D. W. (2000). Breast cancer incidence highest in the range of one species of house mouse, Mus domesticus. British Journal of Cancer, 82(2), 446-451. Apud Lawson, J. S. et al., 2018.

Vieira, S. \& Hossne, W. S. (2015). Metodologia Científica para a Área da Saúde. Elsevier.

Wang, Y., Holland, J. F., Bleiweiss, I. J., Melana, S., Liu, X., Pelisson, I., Cantarella, A., Stellrecht, K., Mani, S. \& Pogo, B. G-T. (1995). Detection of mammary tumor virus env gene-like sequences in human breast cancer. Cancer Research, 55(22), 5173-5179. Apud Lawson, J. S. et al., 2018.

Zammarchi, F., Pistello, M., Piersigilli, A., Murr, R., Cristofano, C. D., Naccarato, A. G. \& Bevilacqua, G. (2006). MMTV-like sequences in human breast cancer: a fluorescent PCR/ laser microdissection approach. The Journal of Pathology, 209(4), 436-444. Apud Lawson, J. S. et al., 2018. 